\title{
Preface to the Special Issue for Sustainable Environmental Technologies
}

Asia Pacific Conference on Sustainable Energy \& Environmental Technologies (APCSEET) has consistently attracted the top professionals in Asia-Pacific region to share their knowledge for the sustainable development since the first conference in 1996 at Singapore. The ninth APCSEET was successfully held on 5-8 July, 2013 at Narita, Japan, by the support of the Society of Chemical Engineers Japan (SCEJ) and other academic societies, universities, and public sectors.

It is our great pleasure to publish here a special issue of the Journal of Chemical Engineering of Japan entitled "Sustainable Environmental Technologies" which consists of one journal review and 10 research papers selected from those presented in APCSEET 2013.

This special issue has been planned by the Division of Environment of SCEJ and handled by the guest editors belonging to this division. We believe that this special issue stimulates the readers of the journal and promotes the research activities related to environmental technologies.

We deeply appreciate the authors of the papers and the peer reviewers for giving us thoughtful and constructive comments. We also thank Prof. Toshinori Kojima, Seikei University, for his effort to organize APCSEET 2013 and his helpful advice on planning this special issue and Ms. Kazuko Yamashita, in the editorial office, for her assistance during the editing process.

Chief Editor for Special Issue

Mikiya Tanaka, National Institute of Advanced Industrial Science and Technology

Editors for Special Issue Akihiro Yamasaki, Seikei University Satoshi Nakai, Hiroshima University Nobuhide Takahashi, Shinshu University

Yuji Sakai, Kogakuin University Satoshi Kodama, Tokyo Institute of Technology 